\title{
EVOLUCIÓN Y SIMBIOSIS DE LAS PROPENSIONES ESENCIALES EN EL ESCENARIO DE LA ENSEÑANZA DE LAS CIENCIAS EXPERIMENTA.LES
}

SAULEDA PARÉS, N. y MARTÍNEZ RUIZ, M.A.

EU Profesorado. Universidad de Alicante. Apartado 99. Alicante.

\section{SUMMARY}

The main prospects on science teaching are analyzed. The recent paradig ns in computer technology bave enabied the development of a second generation of instructional design. Additionally, breakthroughs in neuroscience provide a new focus to ecology in the field of science teaching in the general scenario defined by the theory of evolution. The authors have the conviction that the time has arrived for a new curriculun in sciences based on a symbiosis between computer technology, instructional design and neurobiology. As a consequence, curricular projects in sciences must be especially centered upon neuronal education. Finally, the incidence of the science of complexity and the concept edge of chaos in education is considered.

\section{INTRODUCCIÓN}

El cerebro, merced a su plasticidad, evoluciona anatómica y dinámicamente (Ramón y Cajal 1941).

En un estilo impresionista es posible esbozar con una serie de trazos aproximados el siguiente escenario y sus cambios en el tiempo. La evolución del universo se inició hace unos quince mil millones de años. El universo estaba comprimido en una pequeña región y una explosión, el big bang, originó una nube de energía y materia. El universo se expandió, se enfrió, las estrellas y galaxias coalescieron, y tomó forma el cosmos. La evolución prosiguió y hace unos tres mil quinientos millones de años la vida emergió, probablemente, de simples reacciones químicas: El RNA, con su propiedad de hacer replicas de sí mismo, se formó de ribosa, bases nitrogenadas y ácido fosfórico. Posteriormente, con la cooperación de las proteínas, el RNA adquirió la posibilidad de hacer copias de dos filamentos similares a él, que evolucionaron hacia el DNA. Intermitentemente y en diferentes esca* las, los seres vivos han continuado evolucionando a través de un proceso de selección sobre la información genética codificada en los áciđos nucleicos.

El proceso de la evolución es el responsable de la construcción y riqueza de la biodiversidad. En una línea filogenética de dicho proceso, la neocorteza cerebral se ha ido expandiendo y los hominoides y los homínidos han evolucionado hacia cerebros más complejos con mayor capacidad de procesamiento. El desarrollo de la postura erecta fue un evento crucial en la evolución. El esqueieto de Lucy y otros Australopithecus sugieren que el bipedisnio y las herramientas marcaron el ascenso a la vida cogniliva. El cerebro y el hombre son resultado de la evolución biológica y social y las actividades mentales individuales pueden situarse en un cuadro evolucionista más amplio que incluye cultura e historia (Brunner 1971).

La evolución es creadora. La evolución física propició ta evolución jiológica y la evolución biológica generó la evolución social y cultural, emergiendo los humanos. Ahora, la evolución cultural ha conducido a la evolución genética dirigida por el hombre. Los bioquímicos, incluso, dominan Iil evolución darwiniana a escala molecular y son capaces a través de la mutación, amplificación y selección, de hacer evolucionar conjuntos de macromoléculas havia un objetivo específico (Joyce 1992). EI hombre há pasado, una vez más, de ser peón a ser jugador. Hay una lógica unificadora en la naturaleza: su paradigma para la construcción. No obstante, cliando se asciende en niveles de complejidad, desde los quarks hasta las scriedades humanas, aparecen propiedades que no pueden ser deducidas, ni predichas de las propiedades de los niveles inferiores. El resultado es mucho más que 
la suma de sus partes. La evolución va de lo simple a lo complejo, de las estructuras indiferenciadas a las diferenciadas, aumentando la organización del universo a medida que el tiempo se disipa (Prigogine y Stengers 1984).

Una sociedad se caracteriza principalmente por las tecnologías de la comunicación de que dispone (Vattimo 1990). La era de la información se basa en los ordenadores y las redes que los conectan. La comunicación hace la diferencia, y la tecnología informática aumenta en varios órdenes de magnitud las posibilidades de construcción de significados y de compartir sistemas simbólicos (Bruner 1991). La acelerada evolución de la tecnología informática, electrónica y de las comunicaciones ha llevado a Tesler (1991) a definir para el corto lapso de tiempo que va de los años 60 a los 90 la sucesión de cuatro paradigmas: procesamiento por lotes, tiempo compartido, sobremesa y red (Sauleda y Martínez 1993). Esta alta dinámica evolutiva demuestra claramente la creciente influencia de las tecnologías de la computación, en casi todas las dimensiones de nuesta sociedad y cultura. Consecuentemente, la resolución de los problemas educativos debe superar las fórmulas tradicionales e integrar el beneficio de las actuales tecnologías. En el ámbito de la educación se ha señalado como uno de los factores básicos de la enseñanza el maximizar la comunicación entre profesor y alumno (Silcock 1993). Paradójicamente, la informática puede favorecer esta maximización a través de la ex* ploración, la comunicación, la integración disciplinar, la variación curricular y la autonomía de profesores y alumnos (Salomon 1991). En este escenario coparticipamos de la visión general de Branson (1990), que postula la evolución hacia un paradigma instruccional basado en la tecnología informática y de las comunicaciones. Este paradigma se centra en el alumno y en el uso de Ia tecnología y en un nuevo desarrollo instruccional. El ordenador, casi una mente sin biología, puede ser un compañero de trabajo que debe ser integrado en forma significativa y nuclear en la enseñanza de las ciencias.

Margulis (1992) destaca que, en la evolución de las especies, las asociaciones biológicas -desde el mutualismo hasta el parasitismo- constituyen una poderosa fuerza creativa. La autora defiende que con la simbiosis los organismos pueden combinar sus dotaciones genéticas y conseguir mayores logros que los de cada especie aistada y propone que la selección natural actúa como el editor, pero que no es la fuerza creadora de la evolución. Entre profusos casos demostrativos es de señalar el modelo del origen endosimbiótico de la célula eucariota, que se interpreta en el sentido de que las mitocondrias y cloroplastos proceden de bacterias que fueron incorpo* radas, lo que evitó que los eucariotas tuvieran que redescubrir la respiración y la fotosíntesis.

La evidencia y trascendencia de la fuerza editora y creadora de la simbiosis debe ser fundamento en el camino de provocar la evolución hacia una alianza intencionada entre cerebro, ordenador y educación. La colaboración con los ordenadores supone la existencia de una división complementaria del trabajo, que se hace interdependiente y que se desarrolla en el tiempo (Sauleda 1992). En dicha simbiosis mutualista, el hombre es cada día más dependiente de los ordenadores como es comprobable en los programas científicos espaciales, en la investigación del genoma, en usos médicos, en protección ambiental o en astronomía. Las personas más la tecnología pueden realizar tareas que previamente les resultaban imposibles. La tarea es ahora vincular sistemas que se han vernido desarrollando con una cierta independencia y constituir en un proceso de fulguración (Popper y Lorenz 1992) un sistema con propiedades completamente nuevas. En el marco de la evolución, que da sentido glohal a todo, la humanidad está consiguiendo cada día máquinas más inteligentes. Ahora el objetivo de Ia instrucción is lograr que estas máquinas contribuyan al desarrollo de las aptitudes específicas y de la inteligencia en general de las personas y para ello es preciso adaptar el diseño instruccional a las potencialidades de ta tecnología informática interactiva y a los nuevos modelos científicos del cerebro que aportan la neurobiología y la inteligencia artificial (IA).

Es, justamente, en la enseñanza de las ciencias donde es más conveniente que los énfasis y las tendencias de progresión dimanen de los nuevos modelos científicos del cerebro y de la incorporación de las tecnologías informáticas al diseño instruccional y al aula. Se asume, además, la necesidad de incorporar las más recientes investigaciones de las areas de psicología, lingüística y otros campos científicos. Los centros de desarrollo en la enseñanza de las ciencias han ido evolucionando desde las lecciones de cosas, pasando por la higiene, la biología molecular, el "método científico», la educación sexual, la educación ambiental, la educación para la salud, etcétera. Ahora y aquí, los proyectos a desarrollar deben incidir en la erıseñanza sobre y con las tecnologías interactivas y la :ormación en el conocimiento científico del cerebro y en el uso más efectivo y eficiente del mismo. Sobre este presupuesto general se va a proceder a analizar los caminos a andar y el conveniente lugar de encuentro y comunión de los mismos.

\section{PROPENSIONES EN LA EVOLUCIÓN DEL DISENO INSTRUCCIONAL}

Desde la asuncicin convencida de que las tecnologías interactivas van c. representar una significativa parte de la educación que la sociedad demanda, resulta evidente la conveniencia ce desarrollar una metodología y unos instrumentos que orienten el diseño y desarrollo de materiales de alıa valía y validez basados en dicha tecnología interactiva. La coevolución de la simbiosis insirucción - tecrologías informáticas está definiendo una filogénesis cue va de la enseñanza asistida por ordenador (EAO) hacia la enseñanza inteligentemente asistida por ordenador (EIAO), y de ésta en dirección a la segunda generación de diseño instruccional (DI $)$. Las propensiones que diferencian a la EIAO de Ja EAO pasan por el intento de cue el alumno dirija su propia formación y el ordenaclor desempeñe el papel de un tutor inteligente. El núcleo de la acción tutora depende de un sistema experto e: la materia, de un sistema experto instruccional, y de un gestor del modelo de alumno y de interfaces. El enfoque de dicha instrucción busca la 
optimizáción de la atención, la minimización del tiempo perdido y el favorecer el sentido de control del alumno sobre sus actividades. La EIAO no implica un único modelo didáctico; al contrario, asume la necesidad de una diversidad de estilos de aprendizaje. En dicha enseñanza los programas didácticos tienden a demandar el análisis de los contenidos, Los prerrequisitos, los errores conceptuales o las ideas previas y el tratamiento de estas dimensiones. Consiguientemente, la EIAO, al proponer el ordenador a los alumnos una línea adaptada que éstos siguen tomando sus propias decisiones y al facilitar la superación del escollo del tiempo que exige el diagnóstico inicial de los alumnos y la remediación de las situaciones de aprendizaje (Woerner, Rivers y Vockell 1991), puede ser una respuesta efectiva al problema de optimizar los enfoques basados en los prerrequisitos (Gagné 1985), en los errores conceptuales o preconcepciones de los alumnos (Carrascosa y Gil 1982, Osborne y Wittrock 1983, Driver 1988 1989) oen la resolución de problemas (Polya 1957. Gil et al. 1985 1991, Barrón 1991). El modelo de alumno es un clemento básico de estos sistemas y la situación ideal ss que el modelo atienda a los conocimientos conceptuales, a las características individuales y a las estrategias metacognitivas que domina el aprendiz (Thomipson 1991). Los sistemas de formación en EIAO son diversos. Hanson (1992) distingue entre: los sistemas expertos con simulaciones de casos y explicación del razonamiento de un experto, programas de resolución de problemas, tutores inteligentes, micromundos y simuladores.

No obstante lo esbozado en el párrafo anterior, Merrill y otros (1990) encuentran limitaciones a los sistemas tutoriales inteligentes debido a que no están insertos en el marco de una teoría general de la instrucción. Por otra parte. en el intento de dar un paso más adelante en el camino de la generalización, los citados autores aseveran que los modelos de la primera generación de diseño instruccional (DI, )-teorías y metodologías de Gagné y otros (1970)-comparten una serie de limitaciones: el análisis de contenidos se focaliza en componentes y no en todos integrados; las prescripciones para la adquisición de conocimiento y organización del curso son escasas y superficiales; las teorías son, básicamente, sistemas cerrados; el diseño es a menudo pasivo y no interactivo. En conclusión, estos autores enuncian que los métodos del DI, no consideran la naturaleza altamente interactiva de las nuevas tecnologías y el cómo prescribir secuencias de gran interactividad, debido a que estas teorias son anteriores al desarrollo de la tecnología informática. Para Merril y otros (1990), la suma de limitaciones antes enunciadas demanda cl desarrollo de una segunda generación de diseño instruccional $\left(\mathrm{DI}_{2}\right)$, que dé respuesta al proceso de analizar y guiar la enseñanza de conjuntos integrados de conocimientos y destrezas; que sea capaz de poder producir prescripciones para la sclección de estrategias instruccionales interactivas; que sea un sistema abierto con capacidad de incorporar conocimientos y aplicarlos en el proceso de diseño; $y$, por último, que integre las fases del desarrollo instruccional.

El concepto de la DI, es cognitivo. Merril y otros (1990) asumen que el aprendizaje produce la organización de la mernoria en modelos mentales y, en consecuencia, adoptan las proposiciones de que la organización y la elaboración del conocimiento durante el aprendizaje facilita la posterior recuperación del mismo. Asimismo, consideran que el conocimiento puede ser representado en una base de conocimientos externa al aprendiz; no obstante, no entran en cómo la estructura cognitiva es organizada y elaborada, pero presumen que hay una cierta correspondencia entre la organización y las representaciones de la mente. Nosotros asumimos que el encuentro entre neurociencias, psicología e IA está aportando modelos de suficiente validez para ser integrados en lo que debe dar emergencia a la tercera generación de diseño instruccional $\left(\mathrm{DI}_{3}\right)$, modelos que posibilitarán una radical revolución científica en la instrucción.

En sucesivos artículos, Merril y otros (1991, 1992), en el marco del escenario antes definido, desarrollan la Instructional Transaction Theory. Las transacciones son definidas como algoritmos instruccionales, modelos de interacciones mutuas y en tiempo real entre el sistema y el alumno con un intercambio de información, que son diseñadas para que el estudiante aprenda un conocimiento o destreza. Las transacciones son programadas una sola vezen instructional transaction shells, pudiendo ser usadas con distintos contenidos de similar tipo de conocimientos y destrezas.

Una transaction shell consta de los siguientes componentes primarios: 1) Interacciones y un gestor de interacciones, que es responsable de la puesta en acción de Ias interacciones. Conviene que las transacciones faciliten el control de las interacciones por el alumno o por el sistema y que puedan responder a una estrategia de indagación o expositiva. En general, una transacción se compone de una serie de presentaciones y reacciones, y esta secuencia de interacciones es lo que sus autores denominan una estrategia de interacción, de las que se definen siete tipos distintos: panorámica global, familiarización, dominio básico, dominio completo o experto, remediación básica, remediación completa o experta y evaluación. 2) Parámetros instruccionales que permiten diseñar la instrucción en función de las característicus de los alumnos, de la tarea de aprendizaje y del contexto. Un sistema de autor de configuración de la instrucción permite al diseñador suministrar los valores adecuados de los parámetros instruccionales. 3) Una base de conocimientos que almacena una representación de la totalidad de los conocimientos de aprendizaje. Un sistema de autor de adquisición de conocimientos permite a un experto en la materia enseffar a estructurar el conocimiento a ser enseñado. El conocimiento se representa en objetos que denominan frames, con slots que contienen valores para la estructura y para las relaciones con otros frames. Un frame se organiza en un diagrama en forma de árbol, reuniendose las partes de la entidad en clusters o constelaciones. Las part frames contienen slots para el nombre de la parte de la entidad, las propiedades asociadas e indicadores para los recursos asociados con el frame. 4) Una base de datos de recursos con mediaciones de las representaciones a enseñar. Un sistema de autor editor de recursos facilita la creación de mediaciones de las representaciones del conocimiento. 
Los sistemas de autor son sistemas expertos inteligentes basados en el ordenador que a través de una serie de reglas convierten la información en una serie de valores por defecto en función de los parámetros de la transacción shell. EI sistema es abierto, numerosas decisiones se toman por un conjunto de miniexpertos pedagógicos, buscando que dichas decisiones individuales se coordinen para tomar las grandes decisiones del proceso. En el momento en que la investigacion aporte un nuevo conocimiento que pueda expresarse en una regla en un miniexperto, este conocimiento puede ser incorporado en el sistema. Esta dimensión confiere un carácter evolutivo. El sistema, además, es integrađo y los cambios que se efectúan en una área fluyen y determinan cambios apropiados en otras áreas; de esta forma el sistema autocontrola su coherencia y su carácter completivo. Las transaction shells incorporan inteligencia ya que, para cada tipo de conocimiento, se seleccionan los tipos de interacciones más adecuadas; por otra parte, la base de conocimientos incorpora una sintaxis que garantiza que el conocimiento que se incluya sea consistente.

Merrill y colaboradores (1992) entienden que el diseño que ellos detallan puede parecer más complejo que la real utilización de esta tecnología de la instrucción, ya que, una vez diseñado su uso, responde al modelo de una shell. En el análisis de esta teoría, Gagné y Merrill (1991a, y 1991b) analizan el problema de que si un modelo instruccional es demasiado complejo pierde su valía y coinciden en que hay un cierto nivel de complejidad que resulta confortable para el profesor y que está por ver si el diseño de la teoría de Merrill et al. (1990 1991 y 1992) supera o no dicho nivel. Nosotros entendemos que el diseño de una segunda generación de diseño instruccional es un paso que se debe dar y que el esfuerzo de desarrollar un diseño instruccional con un enfoque hoÍstico y con sistemas expertos informatizados que facilitan el funcionamiento y la evolución del sistema resulta altamente positivo. En cualquier caso, varios de los desarrollos parciales del diseño son de un gran interés teórico y de posible aplicación. En última instancia será Ia experimentación real del modelo lo que informará con más finura de las complejidades, limitaciones y potencialidades, siendo previsible una rápida evolución de la teoría motivada por su novedad y por la nueva tecnología que está en el horizonte más inmediato. Las sucesivas aproximaciones al modelo son las que definirán las dimensiones a perfeccionar y los aspectos excesivamente complejos y problemáticos que deben quedar menos informatizados. En todo caso, el control del profesor sobre el proceso debe estar garantizado y es éste el que debe tomar las decisiones esenciales.

\section{PROPENSIONES EN EL ÁMBITO DE LA NEUROBIOLOGÍA DEL APRENDIZAJE}

La evolución por selección natural (Darwin 1859) es una de las grandes generalizaciones de la historia natural. La evolución es entendida en formas muy diferentes pero hay una residencia común en un amplio darwinismo. En el seno de esta residencia y a partir de la visión de James (1890) de la consciencia como un proceso y del cerebro como un órgario en equilibrio interno inestable en el que los estados de la conciencia son personales, continuos, intencionales v selectivos, Changeux (1992) ha analizado los avances en neurología y ha reconceptualizado el paradigma evalucionista a los niveles cognitivos. Este autor señala cue en cada nivel -átomos, moléculas, neuronas, circuitos, «entendimiento» (grupos de neuronas...) y «razón» (encadenamiento de grupos...)- interviene una forma de retención selectiva con unos mecanismos que producen variaciones o generador de diversidad, unos procesos te selección y unos mecanismos de conservación y transmisión de las variaciones seleccionadas.

La generación de la diversidad viene posibilitada por la complejidad del cerebro, que con sus cien mil millones de neuronas es el objeto más complejo del universo conocido. La diversidad se materializa en la variedad de tipos de neuroras, en las ramificaciones y sinapsis de estas células, y en la diferenciación molecular. En suma, la diversidad estructural, funcional y molecular del cerebro proporcicna una plataforma suficiente para la función mental. Adicionalmente, conviene tomar también en consideración una dimensión más: la plasticidad que poseen las sinapisis como consecuencia de la actividad cerebral. Esta p;asticidad incrementa la complejidad y establece un susirato aún más diverso para las funciones cognitivas y mentales (Fischbach 1992).

En cuanto a los procesos de selección, Changeux y otros $(1983,1992)$ proponen un mecanismo de estabilización selectiva. La actividad de una sinapsis acelera la eliminación de las terminaciones sobrenumerarias. Así, por ejemplo, durante el desarrollo de la inervación de las células de Purkin ie del cerebelo, por las fibras trepadoras tiene Iugar una restricción del número de contactos sinápticos. Las cınexiones sinápticas vienen, en parte, genéticamente predeterminadas y dependen, asimismo, de la competencia en la que se seleccionan las que están. suficientemente activadas. En algunos casos se ha visto que las sinapsis que se usan simultáneamente activan el receptor de NMI)A (el N-metil D-aspartato se une a estos receptores) y se fortalecen, mientras que las que son poco estimulaclas son podadas. El input efectivo puede ser estabilizado, pero previene el desarroilo de otros inputs correlacionados débilmente, generándose una disminución de la plasticidad cerebral. Además, todas las neuronas tienıın la misma dotación genética pero expresan únicame:rte un pequeño subconjunto de genes. La expresión selectiva de genes se ha evidenciado en et seno de poblaciones tan homogéneas como las células amacrinas de la retina, las células de Purkinje en el cerebelo y las netronas motrices de la médula espinal. Asimismo, se ha de mostrado que la actividad de impulso refuerza Ia expresión de genes que codifican factores troficos necesarios para la supervivencia de neuronas. También es bien reconocido el hecho de que se forman más neuronas que las necesarias en el desarrollo y numerosas células mueren por la ausencia de un factor trófico.

A los niveles cogni:ivos, Changeux (1992) selecciona y reformula la propuesta de Dennett (1991) fundamentada en un un modelo evolutivo de selección entre copias múltiples que obran conjuntamente, en el que las opera- 
ciones cognitivas responden a unos procesos multipistas de interpretación y de elaboración que se producen en milisegundos, sobre los que se producen selecciones a través de un proceso de evaluación de la experiencia. El modelo multipistas de Ia consciencia puede explicar la emergencia de ideas nuevas y la creatividad como resultado de la combinación de pistas y la selección de nuevas combinaciones de elementos disjuntos. Las prerrepresentaciones más adecuadas al mundo exterior son seleccionadas progresivamente. La sucesión en el tiempo de ensayos y errores lleva a sistematizaciones. Damasio y Damasio (1992), y Crick y Koch (1992) mantienen que las oscilaciones sincrónicas y coherentes de las neuronas explican los procesos de la consciencia. Las oscilaciones sincrónicas a través de las poblaciones neurales (Yuste, Peinado y Katz 1992) constituyen un importante principio organizador del procesamiento de la información y una noción unificadora.

Changetux (1992) ha diseñado y construido un modelo infornático de arquitectura neural capaz de superar el test de Wisconsin, que valora la capacidad de descubrir reglas en la selección de cartas que tienen símbolos distintos. Modelos de neuronas codifican una representación, por ejemplo una regla de clasificación. Los grupos de neuronas se distribuyen en un nivel sensoriomotor, y un nivel homólogo al de la razón. Hay neturonas de memoria y neuronas-reglas. El organismo utiliza éstas para ensayar una regla de conducta, lo que generará una señal que determinará consonancia o disonancia en función de la respuesta elegida. Algunas neuronas de error son capaces de desestabilizar las neuronas-reglas cuando la respuesta del mundo exterior es disonante. En este caso se producen otras hipótesis de conducta lo que se comporta como un generador de diversidad. El modelo introduce un bucle de autoevaluación. La red evalúa diversas reglas hasta que halla una nueva, mediante un proceso limitado de razonamiento. El modelo actúa por ensayo y error, en función de las señales recibidas del exterior o de las que antes han sido evaluadas y memorizadas, comportándose en armonía con el exterior. El modelo responde a un principio unificador del funcionamiento del cerebro que establece que el córtex compara los inputs con los modelos almacenados y procesa las consecuencias de la comparación en lo que no se adecua con el modelo almacenado. Asimismo, Alkon (1989) ha diseñado redes neuronales artiftciales que, actuando bajo principios biológicos, han sido bastante eficaces en reconocer estructuras, y que además aportan algunas pistas sobre eI funcionamiento de Ia memoria. También Hinton (1992) ha presentado modelos de cómo las redes neuronales artificiales procesan la información y de cómo el cerebro logra aprender.

Edelman (1992) ha reformulado, en su theory of neuronal group selection (TNGS), el esquema de la estabilización selectiva, que ha sido entendido a nivel de mapas neurales, y denominado darwinismo neural. Este atutor postula en su teoría que la selección ocurre a nivel de grupos neuronales y deriva de las fuerzas podadoras de experiencias contingentes. El mecanismo molecular que propone Edelman para la estabilización implica a una clase de glicoproteínas de adhesión celular ( $N$ CAMs), moléculas cuya participación en la memoria ha sido demostrada por diversos grupos. Pero una teoría de la conciencia, o incluso de la memoria, requiere más que mecanismos moleculares, y a ellos Edelman añade un énfasis en lo que llama la «reentrant», conducta de los circuitos neurales; concepto que se supone que consiste en los múltiples feedback y feedforward loops, a través de los cuales grupos de neuronas se ligan en el espacio y en el tiempo. En su búsqueda de experimentar el modelo, ha diseñado un robot, Darwin III, capaz de aprender.

Considerando el cerebro como un sistema activo, dinámico, que se autoorganiza, hay que valorar la propuesta de Popper y Lorenz (1992) de substituir las visiones darwinianas pasivas de la sociobiología por un darwinismo activo en el que el organismo juega un papel en el desarrollo de su propio futuro. En similar dirección Schrödinger (1967) asevera que el comportamiento de los individuos de una especie tiene una influencia muy significativa en la tendencia de la evolución, simulando incluso un falso lamarckismo. Una visión que, como mínimo, resulta bella, estimuladora y vigorizante.

Pensar con el cerebro sobre el cerebro es un proceso sin fin, pero antes de terminar esta disgresión hay que prestar un mínimo de atención a la atención. Brevemente, hay que referir que la atención y la memoria temporal son rasgos claves para la consciencia de los eventos sensibles y, además, que la atención selectiva y la necesidad de dirigir la información selectivamente de un lugar a otro es otro punto nuclear del funcionamiento del cerebro. En todo caso la capacidad de ubicar la necesaria atención a la toma de decisiones es lo que otorga libre albedrío a la especie humana (James 1890).

En suma, la polémica científica sobre el constructivismo (Merrill 1991) y, en general, sobre el aprendizaje debe ser enriquecida por planteamientos más biológicos que recojan la influencia de la evolución, así como los avances en neurobiología y en el campo de la IA. Obviamente, la aproximación evolutiva no lo explica todo, pero entendemos que explica más que otros modelos.

\section{CONCLUSIONES}

En la actualidad no hay una teoría coherente con amplio consenso en la enseñanza de las ciencias, y el metaanálisis resulta problemático (Hurd 1991). Es evidente que un ordenador por sí mismo no modifica la dinámica del aula (Emihovich 1990), la capacidad interactiva de las tecnologías inteligentes, que incluyen tanto la IA como la inteligencia distribuida (Salomon, Perkins y Globerson 1989), está iniciando la génesis de una auténtica revolución educativa. Adicionalmente, aunque tampoco hay una teoría definitiva del cerebro, porque la comprensión del cerebro es difícil por su complejidad y porque es la selección natural la fuerza responsable de su diseño (Fischback 1992), está en curso la revolución neurobiologica. El conocimiento del cerebro es crucial para la supervivencia de la especie humana e inciuso de la del planeta. Por ello, la intencionalidad primera de la educa- 
ción debe ser influir en el binomio cerebro/mente. Las neurociencias y la ciencia de los computadores están construyendo una teoría de lo que significa para el cerebro percibir, aprender y comprenderse a sí mismo (Churchland 1989) y lo que aquí se postula es que los avances en esta teoría sean aplicados extensiva e intensivamente a la enseñanza de las ciencias como contenidos conceptuales, procedimentales y actitudinales.

De la sinergia entre el estado del arte en el campo del diseño instruccional, de las aportaciones en el ámbito de las tecnologías informáticas y de los avances en la formulación de nuevos modelos cognitivos basados en la neurobiología, está emergiendo la plataforma que va a fundamentar la tercera generación de diseño instruccional. La evolución intencionada del proceso de simbiosis entre la educación, la revolución informática y la neurobiológica implicará un gran salto en el acrecentamiento de la efectividad de la tercera generación de diseño instruccional. En dicha dirección se está avanzando a través de sucesivas aproximaciones en un proceso similar al de la acción del cerebro que procesa en paralelo posiblemente porque la selección natural favorece las soluciones rápidas y aproximadas, en vez de las lentas pero exactas.

En la evolución de los sistemas educativos, las mutaciones no surgen al azar y los nuevos equipamientos tecnologicos educativos deben responder a las convicciones e intencionalidades de los expertos y responsables de la educación (Salomon, Perkins y Globerson 1992). El conocimiento del cerebro favorece la comprensión de la percepción, el aprendizaje y otras funciones mentales en términos neurobiológicos, y la IA coadyuva a la comprensión de los principios đe computación. Por ello, es conveniente reformar los contenidos de la enseñanza de las ciencias experimentales de forma que el estudio de la neurobiología sea el núcleo de desarrollo esencial de los proyectos curriculares y que las tecnologías informáticas sean otro polo de desarrollo, además de un recurso metodológico. La selección de las valiosas dimensiones aportadas por los enfoques basados en los errores conceptuales, las preconcepciones de los alumnos y la resolución de problemas han de propiciar que la enseñanza de las ciencias evolucione hacia enfogues más enraizados en la biología, en el conocimiento del cerebro, y en los nuevos modelos propuestos por la IA, así como en las potencialidades de las tecnologías interactivas.

En suma, un currículo de ciencias nucleado en el sistema nervioso y en el cerebro va a entregar una poderosa herramienta a los alumnos con la que se aumentará la potencialidad enunciada por Ramón y Cajal (1941) de que «todo hombre puede ser, si se lo propone, escultor de su propio cerebro». En similar dirección, el citado énfasis favorecerá el fin básico de la enseñanza de las ciencias, que es hacer consciente a los alumnos de cuál es el uso más eficiente del cerebro y de la inteligencia. Además, la formación del profesorado en dicho ámbito facilitará la profundización en el análisis del pensamiento de los profesores (Calderhead y Robson 1991) y a que los profesores generen una interpretación razonada sobre el por qué los alummnos no comprenden (Bachelard 1938). En paralelo con la environmental education o la health education ha llegado el momento de desarrollar Ia neuronal education. No es nada nuevo que el cambio de aproximación en un campo lo transforma. La reforma de los contenidos (Klopfer y Champagne 1990) y el enfoque en la enseñanza de las ciencias elicitará un salto evolutivo que marcará una điferencia con la situación de crisis actual.

En la era de la simpercomputación (Kaufmann y Smarr 1993), la propuesta que se formula pretende no obviar los planteamientos de las ciencias de la complejidad, en el sentido de que ta evolución avanza hacia lo múltiple, hacia la complejidad (Prigogine y Stengers 1984). Es plausible que la evolución derive del hecho de que algunos sistemas complejos tienden hacia el anticaos. La evolución ha llevado los sistemas hacia el orden y quizás a una región en e. borde entre el orden y el caos. Las ciencias de la complejidad defienden que la autoorganización es inevitable en un amplio espectro de sistemas. La teoría del caos establece que hay fenómenos que implican tantos factores que son impredecibles. La complejidad analiza los sistemes que están entre lo predecible y el caos, en el borde intre los dos estados, y un sistema complejo es probable que no esté nunca en equilibrio. La evolución biológicis ocurre en el borde entre el orden y el caos. Si hay demasiado orden, el sistema queda congelado y no puede cambiar, pero, si hay demasiado caos el sistema no guarda memoria alguna de lo que aconteció anteriormente. Las redes en el borde entre orden y caos pueden tener la flexibilidad de adaptarse eficientemente gracias a la acumuleción de variaciones útiles.

Se ha evolucionado hacia una inteligencia más desarrollada, probablemente porque la conducta flexible resulta de sumo valor para la scibrevivencia y la flexibilidad exige procesar informaciór compleja. Si la vida y el cerebro se desarrollan en el edge of chaos, puede ser eficiente abordar la educación como un sistema también en el borde del caos, en ur: espacio ni demasiado estable, ni demasiado caótico. Es problemático evaluar el impacto que va a tener la ciencia de la complejidad (Steward 1992), pero probablemente hoy es una teorfa que no se puede dejar de considerar. Ulteriormente, es de señalar que, si la sociedad evcluciona hacia la complejidad y el caos, es plausible que sea en este caos donde residan las esperanzas de libertad (Vattimo 1990).

Nuestra visión es consciente del alto grado de elusividad de la ciencia en la frontera y por ello se enmarca en la visión y convicción de que la ecología del campo de la enseñanza de las ciencias hace conveniente que se acomoden, compitan y se integrer diferentes proyectos y aproximaciones. Sentimos y creemos que al small is beautiful hay que adicionarle la diferencia es bella. En el marco del postmodernismo, que cree en un mundo de múltiples causas y en el pluralismo feórico, revisitando el darwinismo cultural en el sentido d: que las teorías más adaptadas son las que sobreviven y desde la asunción de ia valía del pragmatismo y del dialogismo (Henderson 1992), es donde hay que ubicar lé educación neuronal, o sea en convivencia crítica con or ras aproximaciones y en alianza con un modelo hondamente humanístico (Cheung y Taylor 1991). En todo caso, sea o no cierta la idea de Darwin de que eI sentido de la belleza es una propiedad 
estética inherente a los sistemas nerviosos de los animales (Enquist y Arak 1993), desde la asunción de que la inteligencia es la más bella de todas las cosas, nos definimos esperanzados en la frontera entre milenios.

\section{REFERENCIAS BIBLIOGRÁFICAS}

ALKON, D.L., 1989. Memory storage and neural systems, Scientific American, 261(1), pp. 26-34.

BACHELARD, G., 1938. La formation de l'esprit scientifique. (Vrin: París).

BARRON RUIZ, A., 1991. Aprendizaje por descubrimiento. Análisis crítico y reconstrucción teórica. (Amarú: Salamanca).

BRANSON, R.K., 1990. Issues in the design of schooling: hanging the paradigm, Educational Technology, 30 (4), pp. $7-10$.

BRUNER, J.S., 1971. Towardatheory of instruction. (The Belknap Press of Harvard University Press: Cambridge. Massachusetts).

BRUNER, J., 1991 . Actos de significado. Másalláde la revolución cognitiva. (Alianza: Madrid).

CALDERHEAD, I., y ROBSON, J., 1991. Images of teaching: Student teachers'early conceptions of classroom practice, Teaching \& Teacher Education, 7 (1), pp. 1-8.

CHANGEUX, J.P., 1983.L homme neuronal. (Arthème Fayard). (Ed. española en Espasa Calpe, 1985).

CHANGEUX, I.P., 1992. Las neuronas de la razón, Mundo científico, 127, pp. 716-725.

CHEUNG, K.C. y TAYLOR, R, 1991. Towards a humanistic constructivist model of science learning: changing perspectives and research implications, Journal Curriculum Studies, 23, pp. 21-40.

CHURCHLAND,P.S., 1989. From Descartes to neural networks, Scientific American, 261(1), p. 100.

CARRASCOSA, J. y GIL, D., 1982. Los errores conceptuales en la enseñanza de la física I. Un estudio de su persistencia. Actas I Jomadas Investigación Didáctica en Física y Química. (ICE Universitat de València: Valencia).

COLL, C. y SOLÉ, I., 1990. La interacción profesor/alummno en el proceso de enseñanza/aprendizaje, en Coll, C., Palacios, J. y Marchesi, A (comp.). Desarrollo psicológico yeducación II. (Alianza Psicología: Madrid).

CRICK, F. y KOCH, C., 1992. The problem of conciousness, Scientific American, 267(3), pp. 152-159.

DAMASIO, A.R. y DAMASIO, H. 1992. Brain and Language, Scientific American, 267(3), pp. 88-109.

DENNETT, D., 1991. Consciousness explained. (Little Brown and Co.).

DARWIN, C., 1859. The Origin of Species. (Numerosas ediciones en español: Grijalbo 1957, Edaf 1965, Bruguera 1967...).

DRIVER, R., 1988. Un enfoque constructivista para el desarrollo del currículo de ciencias, Enseñanza de las Ciencias 6(2), pp. 109-120.

DRIVER, R, 1989. Students' conceptions and the learning of science, International Journal Science Education, 11, pp. 481-489.
EDELMAN, G., 1992. Bright Air, Brilliant Fire: On the Matter of the Mind. (Basic Books/Allen Lane).

EMIHOVICH, C., 1990. Technocentrism revisited: Computer literacy as cultural capital, Theory into practice, 29(4), pp. 227-234.

ENQUIST, M. y ARAK, A., 1993. Selection of exaggerated male traits by fernale aesthetic senses, Nature, 361(6411), pp. 446-448.

FISCHBACH, G.D., 1992. Mind and Brain, Scientific American, 267(3), pp. 48-59.

GAGNÉ, R.M., 1970. The conditions of learning. (Holy, Rinehart y Winston: Nueva York).

GAGNÉ, R.M. y MERRILL, M.D., 1991a. ICAI, Computers in the schools, an Instructional Design Expert System. In Conversation, en Twitchell, D., Educational Technology, 1, pp. 34-41.

GAGNÉ, R.M. y MERRILL, M.D., 1991b. Integrative goals for instructional design, $E T R \& D, 38$, pp. 23-30.

GIL, D. y MARTÍNEZ-TORREGROSA, J., 1985. La resolución de problemas de física. (MEC: Madrid).

GIL, D., CARRASCOSA, I., FURIÓ, C. y MARTÍNEZ TORREGROSA, J., 1991. La enseñanza de las ciencias en la educación secundaria. (ICE. Universitat de Barcelona: Barcelona).

HANSON, M.C. 1992. El ordenador «Pedagogo», Mundo cientifico, I2(129), pp. 928-936

HENDERSON, J.G., 1992. Curriculum discourse and the question of empowerment, Theory into Practice, 31(3), pp. 204-209.

HINTON, G.E., 1992. How Neurał Networks learn from Experience, Scientific American, 267(3), pp. 144-\$1.

HURD, P., 1991. Issues in linking rescarch to science teaching, Science Education, 75(6), pp. 723-732.

IAMES, W., 1890. Text-book of psychology.(MeMilian and Co.).

IOYCE, G.F., 1992. Directed molecular cvolution, Scientific American, 267, pp. 48-55.

KAUFMANN IYI, W.J. y SMARR, L.L., 1993. Supercomputing and the transformation of science. (Scientific American Library: Nueva York).

KLOPFER, L.P. y CHAMPAGNE, A.B., 1990. Ghosts of crisis past, Science Education, 74(2), pp. 133-154.

MARGULIS, L., 1992. Symbiosis incellevolution. (W.H. Freeman; Nueva York).

MERRIL, M.D., 1991. Constructivism and instructional tesign, Educational Technology, 5, pp. 45-53.

MERRIL, D.M.,LI,Z y JONES, M.K., 1990. Second generation instructional design ( $\mathrm{ID}_{2}$ ), Educutional Technology, 2, pp. $7-14$. 
MERRIL, D.M. LI, Z y JONES, M.K., 1991. Instructional transaction theory: an introduction, Educational Technology. 6, pp. 7-12.

MERRIL, D.M., LI, Z. y JONES, M.K., 1992. Instructional Transaction Shells: Responsabilities, methods, and parameters, Educational Technology, pp. 5-25.

OSBORNE, R. y WITTROCK, M.C., 1983. Learning science: a generative process, Science Education, 67 (4), pp. 489-508.

POLYA, G., 1975. Cómo planear y resolver problemas, ed. original, 1957. (Trillas: México)

POPPER, K.R. y LORENZ, K., 1992. El porvenir está abierto. (Tusquets: Barcelona).

PRIGOGINE, I. y STENGERS, I., 1984. Order out of caos. Flamingo. (Collins: Glasgow).

RAMÓN Y CAJAL, S, 1941. Reglas y consejos sobre investigación científica. (Espasa Calpe: Madrid).

SALOMON, G., 1991. From theory to practice: The International Science Classromm - A Technology-Intensive, Exploratory, Team-based and Interdisciplinary High School Project. Learning: New conceptions, New opportunities, Educational Technology, 6, pp. 41-44.

SALOMON, G., PERKINS, D.N. y GLOBERSON, T., 1992. Coparticipando en el conocimiento: la ampliación de la inteligencia humana con las tecnologías inteligentes, Comunicación, Lenguaje y Educación, 13, pp. 6-22.
SAULEDA, N., 1992. Propensiones en la enseñanza de las ciencias. El escenario del proximo milenio, Tossal. Revista Interdepartantental, $1(0)$, pp. 11-23.

SAULEDA, N. y MARTÍNEZ, M.A., 1993. Evaluación de software educativo, Enseñanza. Anuario Interuniversitario.

SCHRÖDINGER, E, 1967. What is life? and Mind and Matter. (Cambridge University Press: Cambridge).

SILCOCK, P., 19*3. Can we teach effective teaching?, Educational Review, 45(1), po. 13-19.

STEWARD, I., 1993. Nature's semantics, Nature, 361(6412), 507.

TESLER, L.G., 1991. Informática de redes para nuestra época, Investigaciór: y Ciencia, 182, pp. 28-36.

THOMPSON, N., 1991. Computers, curriculum and the learning environment Computers \& Education, 16(1), pp. 1-6.

VATTIMO, G., 1990. La sociedad transparente. (Paidós-ICE Universitat futonoma de Barcelona: Barcelona).

WOERNER, I.J., RIVERS, R.H. y VOCKELL, E.L., 1991. The computers in the science curriculum. (McGraw-Hill. Watsonville].

YUSTE, R., PIIINADO, R. y KATZ, L.C., 1992. Neuronal domains in developing neocortex, Science, 257, pp. $665-669$. 
$$
\text { . }
$$ 